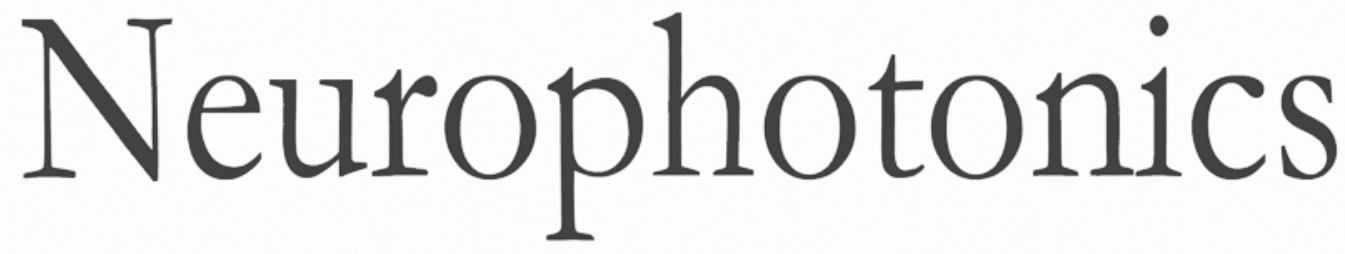

\title{
Differences in orientation tuning between pinwheel and domain neurons in primary visual cortex depend on contrast and size
}

Yong-Jun Liu

Maziar Hashemi-Nezhad

David C. Lyon 


\title{
Differences in orientation tuning between pinwheel and domain neurons in primary visual cortex depend on contrast and size
}

\author{
Yong-Jun Liu, ${ }^{\text {a,b }}$ Maziar Hashemi-Nezhad, ${ }^{\text {a,c,d }}$ and David C. Lyon ${ }^{\text {a,* }}$ \\ anniversity of California Irvine, School of Medicine, Department of Anatomy and Neurobiology, Irvine, California, United States \\ ${ }^{\mathrm{b}}$ Chinese Academy of Agricultural Sciences, Institute of Apicultural Research, Department of Honeybee Protection and Biosafety, Beijing, China \\ ${ }^{\circ}$ Technical University Berlin, Neuroinformatics Group, Department of Software Engineering and Theoretical Computer Science, Administrative \\ Office MAR 5-6, Marchstraße, Berlin, Germany \\ ${ }^{\mathrm{d} B e r n s t e i n ~ C e n t e r ~ f o r ~ C o m p u t a t i o n a l ~ N e u r o s c i e n c e, ~ B e r l i n, ~ G e r m a n y ~}$
}

\begin{abstract}
Intrinsic signal optical imaging reveals a highly modular map of orientation preference in the primary visual cortex (V1) of several species. This orientation map is characterized by domains and pinwheels where local circuitry is either more or less orientation selective, respectively. It has now been repeatedly demonstrated that neurons in pinwheels tend to be more broadly tuned to orientation, likely due to the broad range of orientation preference of the neighboring neurons forming pinwheels. However, certain stimulus conditions, such as a decrease in contrast or an increase in size, significantly sharpen tuning widths of V1 neurons. Here, we find that pinwheel neuron tuning widths are broader than domain neurons only for high contrast, optimally sized stimuli, conditions that maximize excitation through feedforward, and local cortical processing. When contrast was lowered or size increased, orientation tuning width sharpened and became equal. These latter conditions are conducive to less local excitation either through lower feedforward drive or by surround suppression arising from long-range cortical circuits. Tuning width differences between pinwheel and domain neurons likely arise through more local circuitry and are overcome through recruitment of longer-range cortical circuits. ๑ 2017 Society of Photo-Optical Instrumentation Engineers (SPIE) [DOI: 10.1117/1.NPh.4.3.031209]
\end{abstract}

Keywords: contrast invariance; feedback; horizontal connections; optical imaging; orientation map; orientation selectivity; surround suppression; V1.

Paper 16070SSR received Dec. 5, 2016; accepted for publication Apr. 24, 2017; published online May 13, 2017.

\section{Introduction}

The landmark technique of intrinsic signal optical imaging developed by Grinvald et al. ${ }^{1,2}$ has led to a greater understanding of brain organization and function across a diverse array of animal models. ${ }^{3-16}$ Particularly important to visual neuroscience was the revealing of orientation preference maps in cat visual cortex. This allowed for highly accurate targeting of orientation columns, referred to as orientation domains, and also revealed the presence of so-called pinwheels. ${ }^{17,18}$ Pinwheels are formed by the convergence of neighboring orientation domains to a single point, around which the entire range of orientation preferences are represented within a very short distance. The discovery of this unique organization enabled comparisons between homogeneous (domains) and heterogeneous (pinwheel) regions with respect to the orientation preference of local populations of neurons. ${ }^{19-27}$

Using this technique, our work and the work of others have shown that pinwheels in the primary visual cortex correlate to poorer orientation selectivity of their component neurons as compared to domain regions. ${ }^{28-31}$ This lower selectivity can occur specifically for optimally sized classical receptive field (CRF) stimuli, where the relatively small stimulus size elicits the maximum response, and where the driving mechanisms are largely feedforward and local cortical circuits. ${ }^{28,32-38}$ Thus, the

*Address all correspondence to: David C. Lyon, E-mail: dclyon@uci.edu broader range of orientation preference of the local inputs likely leads to the broader selectivity of pinwheel cells. ${ }^{24,39}$

Other factors, such as the size and contrast of the visual stimulus, are also known to influence the orientation tuning and selectivity of individual V1 neurons. For example, larger sized stimuli incorporating the extraclassical suppressive surround (ECS) exhibit significantly sharper tuning, ${ }^{36,38,40,41}$ through subtractive suppression. ${ }^{42}$ This suppressive surround can lead to contrast invariance of orientation tuning of V1 neurons. Conversely, smaller CRF stimuli, which lack the suppressive surround, often yield contrast variant orientation tuning where tuning width is significantly broader at high contrast (HC) compared to low contrast (LC). ${ }^{36}$

While orientation tuning can vary by stimulus features, such as size and contrast, and by position in the orientation map, it is not yet known whether or how these two factors interact to affect orientation tuning. One basic prediction is that tuning for all conditions, LC and HC of the CRF and ECS would be broader at pinwheels compared to domains. To test this, we first obtained orientation preference maps and then targeted microelectrodes to pinwheel or domain centers to record responses to CRF and ECS stimuli at LC and HC. We found, unexpectedly, that orientation tuning derived from the LC CRF was indistinguishable for pinwheel and domain neurons. In addition, while both domain and pinwheel neuron tuning broadened as expected at 
HC, pinwheel neurons broadened significantly more. When including the ECS, we found that tuning width for pinwheel and domain neurons was again indistinguishable and did not broaden with an increase in contrast.

Our results provide new evidence that orientation tuning width differences between pinwheel and domain neurons depend on $\mathrm{HC}$ CRF conditions, which maximize excitation through feedforward and local cortical processing, ${ }^{33,35,37,43}$ where for pinwheel neurons surrounding local inputs are more broadly tuned compared to domain neurons. ${ }^{24,39}$ Conversely, all other conditions result in less local excitation, either through lower feedforward drive or by engaging longer range cortical circuits involved in surround suppression. ${ }^{32,35,36,37,43-48}$ Therefore, tuning width differences between pinwheel and domain neurons are likely generated through more local circuits and overcome through recruitment of long-range cortical circuits.

\section{Methods}

\subsection{Animal Preparation}

All procedures were approved by the Institutional Animal Care and Use Committee of the University of California, Irvine. The experiments were performed on 10 live adult cats weighing 2 to $5 \mathrm{~kg}$ and of both sexes. Animals were initially anesthetized with a mixture of ketamine $(21 \mathrm{mg} / \mathrm{kg}$, intramuscular) and xylazine ( $3 \mathrm{mg} / \mathrm{kg}$, intramuscular). Tracheal and venous cannulations were then performed and animal anesthesia maintained with isoflurane $(0.2 \%$ to $1.0 \%)$ in a $67: 33$ mixture of nitrous oxide and oxygen. Electroencephalogram (EEG), electrocardiogram (EKG), and expired $\mathrm{CO}_{2}$ were monitored to ensure a proper level of anesthesia throughout the entire experiment. Body temperature was maintained around $37^{\circ} \mathrm{C}$ through a heating pad and homeothermic monitor (Harvard Apparatus, Holliston, Massachusetts). To prevent eye movements, neuromuscular blockade was induced with a bolus of vecuronium bromide $(0.6 \mathrm{mg} / \mathrm{ml}$, intravenous $)$ and then maintained with $0.15 \mathrm{mg} / \mathrm{ml}$ during the whole experiment at a flow rate of $2.0 \mathrm{ml} / \mathrm{kg} / \mathrm{h}$ mixed with dexamethasone $(0.5 \mathrm{mg} / \mathrm{kg} / \mathrm{h}$, intravenous) to reduce inflammation in a solution of $5 \%$ dextrose and lactated Ringer's solution. Air-permeable corrective contact lenses were used to protect corneas, and pupils were dilated and accommodation blocked with $1 \%$ atropine sulfate solution, and the nictitating membranes were retracted with $2.5 \%$ phenylephrine hydrochloride. Corrective lenses were placed in front of the eyes during optical imaging and single-unit recording experiments, if necessary to focus the eyes on a tangent screen $37 \mathrm{~cm}$ away. Artificial pupils $3 \mathrm{~mm}$ in diameter were placed in front of the eyes. The locations of the optic disc and area centralis for each eye were plotted daily with a fiber-optic light source. ${ }^{49}$ A craniotomy was made above the dorsal surface of area 17 (V1), and a 20-mm diameter recording chamber was fixed to the skull with dental cement. The dura was removed, and the chamber was filled with silicon oil and sealed with glass for optical imaging.

\subsection{Visual Display Apparatus}

Visual stimuli were displayed on a 21-in. View Sonic Graphics Series G225f CRT screen ( $\gamma$ calibrated) with a mean luminance of $50 \mathrm{~cd} / \mathrm{m}^{2}$. The monitor refreshed at $100 \mathrm{~Hz}$ in a noninterlaced mode with a spatial resolution of $640 \times 480$ pixels. Stimuli were generated using EXPO software (courtesy of
Dr. Peter Lennie) that runs on a G5 Mac with an ATI Radeon 9200 graphics card.

\subsection{Intrinsic Optical Imaging and Orientation Preference Maps}

\subsubsection{Light source and camera}

The V1 (area 17) surface of the cortex was illuminated by two adjustable light guides that were powered by a 100-W tungsten halogen lamp driven by a stable DC power supply (Kepco Inc., Flushing, New York), the range of the Kepco power output was kept between 8.0 to $9.0 \mathrm{~A}$ and 9.9 to $10.0 \mathrm{~V}$. The light was passed through an interference bandpass filter. The image used for a reference map of the blood vessel pattern on the cortical surface [see Fig. 1(e)] was obtained by green light at $546 \mathrm{~nm}$. The imaged depth was then focused $\sim 400 \mu \mathrm{m}$ below the surface and the intrinsic signals imaged using red light at $605 \pm 10 \mathrm{~nm}$. Intrinsic signal optical images were obtained using Grinvald's differential video acquisition system, the Optical Imager 3001 (Optical Imaging Inc., Rehovot, Israel). Images were captured with a 12-bit video camera (Adimec $1000 \mathrm{M} / \mathrm{D}$, The Netherlands; $>60 \mathrm{~dB}$ signal-to-noise ratio) consisting of a $1004 \times 1004$ array of pixels equipped with a $50 / 50 \mathrm{~mm}$ tandem-lens combination for higher magnification. ${ }^{50}$ It provided a $5 \mathrm{~mm} \times 7 \mathrm{~mm}$ imaging window, though regions of interest were usually smaller and were set prior to imaging. The camera was positioned over the exposed cortex such that the optical axis was perpendicular to the cortical surface.

\subsubsection{Visual stimuli for optical imaging and data acquisition}

Orientation preference maps ${ }^{17,24}$ were obtained by intrinsic signals that were recorded in response to a binocularly viewed full screen, with eight different oriented full-field (22.5 deg $\times 180 \mathrm{deg})$, HC $(100 \%)$ square-wave gratings $(0.2$ to 0.3 cycles / deg) drifting at $2 \mathrm{~Hz}$. To prevent transitory orientation nonspecific responses, each trial started with a 500-ms static grating, followed by 4-s drifting in one direction, and then 4-s drifting in the opposite direction. Each block of trials consisted of eight orientations and one blank stimulus set to the mean background luminance, presented in a pseudorandom order. An 8-s interstimulus interval was used to separate each trial to prevent neuronal habituation during data acquisition. Each block was repeated 40 to 60 times. Single condition maps [Figs. 1(a)-1(d)] for each orientation were derived by integrating each trial block over its six frames first, and then averaging across the repetitions, after which dividing by the average of the blank trials. Pseudocolored orientation preference maps [i.e., Fig. 1(f)] were generated through pixel-by-pixel vector summation of the eight single conditions. ${ }^{17,24,51}$ For each orientation map, masks were created to exclude pixels where the map could not be determined due to bone, major blood vessels, or the convolutions of the cortex.

Recording locations were chosen based on how accurate and reproducible the map locations were. Locations were considered reproducible when no displacement of pinwheel centers occurred between two maps generated from two subsets of four alternate orientations; the first set consisted of orientations $0 \mathrm{deg} / 180 \mathrm{deg}, 45 \mathrm{deg} / 225 \mathrm{deg}, 90 \mathrm{deg} / 270 \mathrm{deg}$, and $135 \mathrm{deg} /$ $315 \mathrm{deg}$, whereas the second consisted of orientations $22.5 \mathrm{deg} /$ $202.5 \mathrm{deg}, 67.5 \mathrm{deg} / 247.5 \mathrm{deg}, 112.5 \mathrm{deg} / 292.5 \mathrm{deg}$, and 

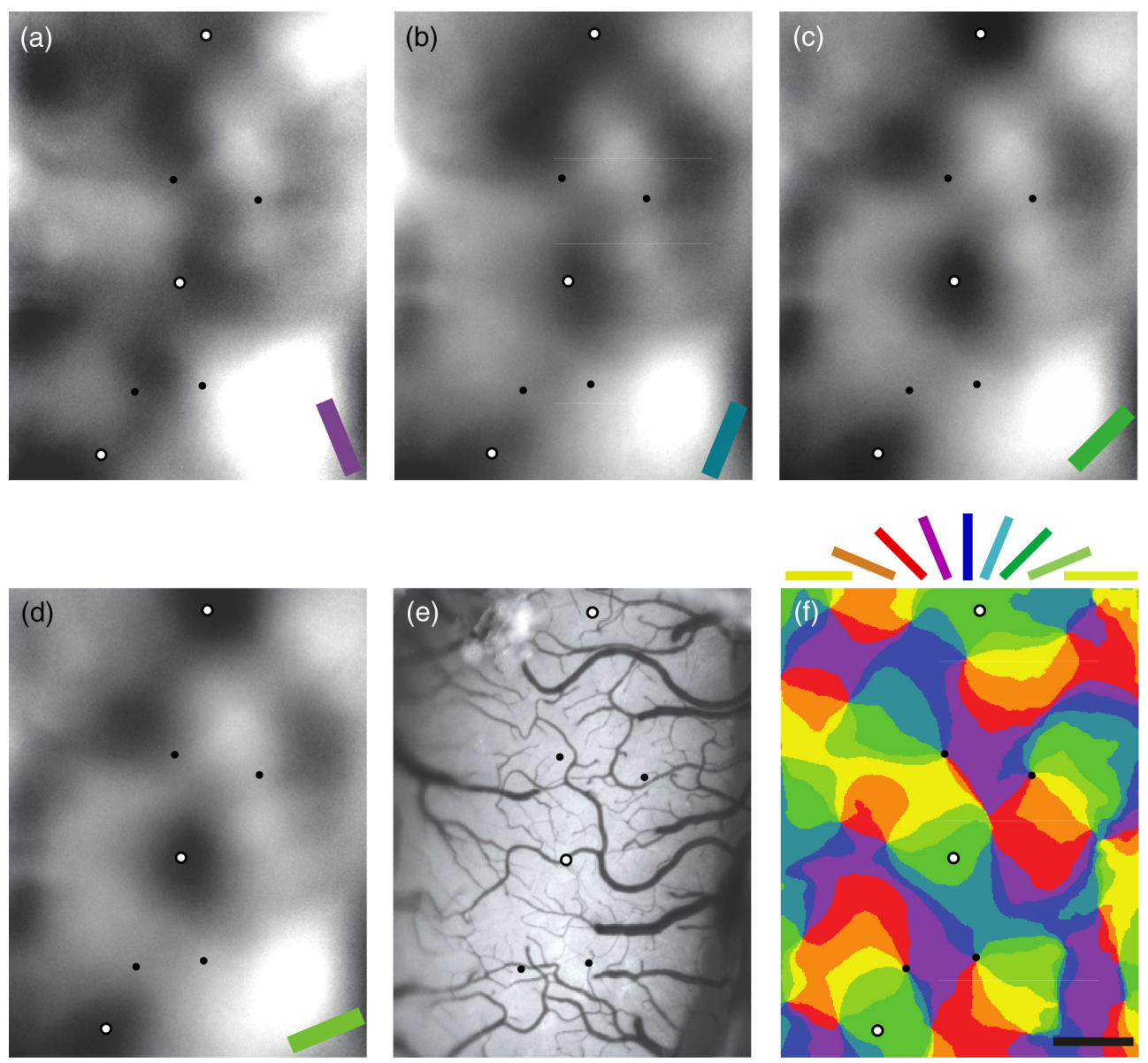

Fig. 1 Intrinsic signal optical imaging of the orientation map on the dorsal surface of a cat's primary visual cortex (V1). (a-d) Example single condition orientation maps are shown. Dark regions indicate areas of high neural activity for stimuli at (a) $112.5 \mathrm{deg} / 292.5 \mathrm{deg}$, (b) $67.5 \mathrm{deg} / 247.5 \mathrm{deg}$, (c) $45 \mathrm{deg} / 225 \mathrm{deg}$, and (d) $22.5 \mathrm{deg} / 202.5 \mathrm{deg}$. (e) Reference image of the surface blood vessel pattern. (f) Orientation preference map. Each orientation domain is color coded at 22.5-deg orientation increments; legend is shown above. Scale bar is $250 \mu \mathrm{m}$. In all panels, the open and solid dots highlight domain and pinwheel sites selected suitable for targeting microelectrodes for single-unit recordings.

$157.5 \mathrm{deg} / 337.5 \mathrm{deg}$. Color maps from the two subsets were compared and only pinwheel sites and domain centers that were congruent between the maps (including the original eight condition maps) were selected for microelectrode recording.

\subsection{Single-Unit Recording}

Single-unit extracellular recordings were made using epoxyinsulated tungsten microelectrodes [3 to $7 \mathrm{M} \Omega$; four-channel amplifier (FHC), Bowdoin, Maine] placed perpendicular to the surface to target either domains or pinwheels. Once inserted below the cortical surface, the brain was covered by a $1.5 \%$ agar solution in saline and sealed with physiological wax to reduce brain pulsation. Electrodes were coated in DiI $(50 \mathrm{mg} / \mathrm{ml})$ prior to penetrating the brain in order to confirm the perpendicularity of our penetrations through histology (see Ref. 28 for more details). Action potentials of isolated single neurons were amplified using an Xcell-3 FHC. Evoked action potentials were broadcast over a loudspeaker for subjective analysis and were sorted with an online window discriminator to isolate single units prior to storing digitally using EXPO signal-processing software.
All cells were stimulated through the dominant eye with the nondominant eye occluded. Once isolated, the cell's receptive field center and preferred orientation were estimated with a 6-deg circular square-wave grating patch. The diameter of the patch was then decreased to $1 \mathrm{deg}$ in the preferred orientation to pinpoint the receptive field center by moving the stimulus systematically under mouse control; similar to methods described elsewhere. ${ }^{28,43}$ To identify optimal stimulus parameters, several visual response properties of the cell were tested in detail. First, the preferred orientation was reassessed by drifting sine-wave gratings oriented in 22.5-deg steps, within a 10-deg circular aperture, a spatial frequency of 0.2 cycles/ deg, and a temporal frequency of $4 \mathrm{~Hz}$ at $100 \%$ contrast. Next, using the preferred orientation and the parameters listed above, preferred spatial and temporal frequencies were determined. Finally, with these optimal parameters the preferred receptive field size for each cell was determined using apertures ranging from $0.2 \mathrm{deg}$ to $30 \mathrm{deg}$ in 18 steps [see Fig. 2(b)].

The CRF was defined from the aperture tuning curve as the aperture size resulting in the greatest mean response, and the ECS was defined as the aperture size greater than the CRF resulting in the lowest mean response [Fig. 2(b)]. Next, using CRF-sized stimuli, contrast tuning profiles were generated 
(a)

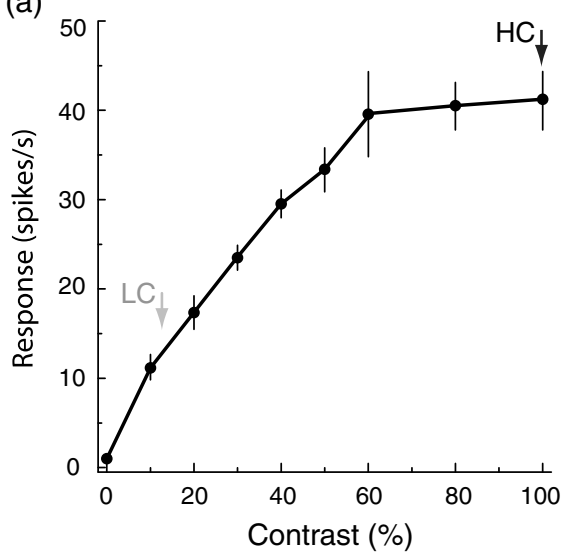

(b)

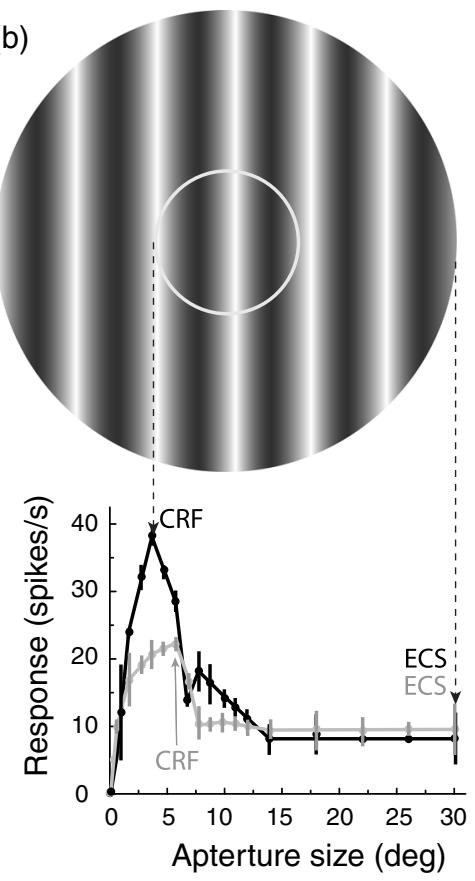

Fig. 2 CRF and ECS identification. (a) For this example neuron, $100 \%$ contrast elicits the maximum response (black arrow) and is chosen as the $\mathrm{HC}$. The percent-contrast eliciting $30 \%$ of the maximum response is $11 \%$ (gray arrow) and is chosen as the LC. (b) Aperture tuning curves at $\mathrm{HC}$ (black trace) and LC (gray trace) are shown. The grating stimulus pictured above indicates the HC CRF (inner white circle) and the HC ECS (outer edge of the whole aperture), with dashed arrows pointing to the corresponding peak response at 3.5 deg and the point of subsequent maximum suppression at 30 deg. Due to LC summation the CRF size at LC is slightly larger, 5 deg (gray arrow), as indicated by the rightward shift of the peak response of the gray tuning curve. The LC ECS is selected as 30 deg.

using nine contrasts, ranging from $0 \%$ to $100 \%$ [see Fig. 2(a)], optimal spatial and temporal frequencies, and the preferred orientation. If the contrast profile saturated before $100 \%$ contrast, the nonsaturating contrast was defined as the $\mathrm{HC}$ and the aperture tuning protocol described above was repeated again to obtain the CRF and ECS at the nonsaturating HC. LC was defined as $30 \%$ of the maximum response. CRF and ECS sizes were then determined at this LC. Orientation tuning profiles were then obtained under optimal spatial and temporal frequencies with $\mathrm{CRF}$ and $\mathrm{CRF}+\mathrm{ECS}$ stimuli shown at $\mathrm{HC}$ and LC. Stimulus sets for each of the four conditions were shown separately, and in different orders for each cell. Stimuli were presented for $2 \mathrm{~s}$ and repeated six times. Each stimulus set used an interstimulus time interval of $2 \mathrm{~s}$, and a blank stimulus with the mean luminance was used to determine the spontaneous activity.

\subsection{Data Analysis}

\subsubsection{Orientation tuning width}

The orientation responses were fitted by the sum of two Gaussians $^{52}$ using

$\mathrm{R}_{\mathrm{O}_{\mathrm{s}}}=$ baseline $+\mathrm{R}_{\mathrm{p}} \mathrm{e}^{-\left(\mathrm{O}_{\mathrm{s}}-\mathrm{O}_{\mathrm{p}}\right)^{2} /\left(2 \sigma^{2}\right)}+\mathrm{R}_{\mathrm{n}} \mathrm{e}^{-\left(\mathrm{O}_{\mathrm{s}}-\mathrm{O}_{\mathrm{P}}+180\right)^{2} /\left(2 \sigma^{2}\right)}$,

where $\mathrm{O}_{\mathrm{s}}$ is the stimulus orientation, $\mathrm{R}_{\mathrm{O}_{\mathrm{s}}}$ is the response to different orientations, $R_{o}$ is the spontaneous response, $\mathrm{O}_{p}$ is the preferred orientation, $R_{p}$ and $R_{n}$ are the responses at the preferred and null orientations, respectively, and $\sigma$ is the tuning width.

Orientation tuning bandwidth of each tuning curve was defined as the half-width at half-height (HWHH), which equals $1.18 * \sigma$ based on the above equation. Our orientation tuning curves typically had two peaks, one for each direction over 360 deg. For each cell, we chose the higher of the two peaks under the CRF HC condition, which was always the condition yielding the maximum response, and used $\mathrm{HWHH}$ for this direction across the other three conditions. Fits were made without subtracting spontaneous activity similar to the procedures of Ref. 53.

\subsubsection{Coefficient of determination, $R^{2}$}

The coefficient of determination was used to evaluate the goodness of our fits

$R^{2}=\left[1-\sum_{j=1}^{N}\left(R_{n}-F_{n}\right)^{2} / \sum_{j=1}^{N}\left(R_{n}-R_{0}\right)^{2}\right]$,

where $R_{n}$ is the response to the $j$ 'th stimulus, $F_{n}$ is the predicted value, and $R_{0}$ is the mean response of the actual data. The mean $R^{2}$ for all of our fits is $0.85 \pm 0.01$.

\subsubsection{Homogeneity index}

The homogeneity of orientation preference of the local environment for each recording site on the orientation map was calculated as in Refs. 24 and 31. For the orientation preference maps, the homogeneity index (HI) for a cortical location $x$ is defined as

$$
\mathrm{HI}(x)=\frac{1}{2 \pi \sigma^{2}}\left|\int \exp \left(\frac{-\|x-y\|^{2}}{2 \sigma^{2}}\right) \exp \left(i 2 \theta_{y}\right) \mathrm{d} y\right|,
$$

where $\theta_{y}$ is the orientation preference at site $y$ and $\sigma$ determines the spread of the spatial scale. We used a value of $\sigma=180 \mu \mathrm{m}$ to match the typical spread of basal dendrites of V1 neurons. ${ }^{31}$ An index value of 1 indicates that the local neighborhood of neurons within $180 \mu \mathrm{m}$ is completely homogenous in their orientation preferences (approximately the domain center), while a value of 0 indicates complete orientation heterogeneity (approximately the pinwheel center). 


\subsubsection{Circular variance}

In addition to HWHH, orientation selectivity was measured more globally using circular variance $(\mathrm{CV})$, which was calculated by Ref. 54

$\mathrm{CV}=1-\sum_{n} R_{n} \exp \left(i 2 \theta_{n}\right)$

where the angle $\theta_{n}$ is the orientation of the $n$ 'th stimulus in radians and $R_{n}$ is the mean firing rate at orientation $\theta$. For these calculations, the spontaneous activity was removed. CV values approaching 0 indicate higher selectivity.

\subsubsection{Significance Tests}

Prior to statistical analysis of groups, we tested whether or not distributions were normal using Lilliefors test. The distribution of HWHH values was not normal; therefore, a Mann-Whitney $\mathrm{U}$ test was used when making these comparisons. On the other hand, the distributions of CV values were not statistically different from normal; therefore, $t$-tests were used for comparisons. The distributions of CRF and ECS sizes calculated at HC and LC were also normal and therefore $t$-tests were used for comparisons.

\section{Results}

To examine how conditions for contrast variance or contrast invariance affect orientation tuning of cells at different positions in the V1 orientation map, we recorded from 140 isolated neurons located in the centers of domains $(n=86)$ or pinwheels $(n=54)$. We have previously shown that limiting stimuli to a cell's CRF is conducive to contrast variance; whereas the addition of the ECS is more conducive to contrast invariance. ${ }^{36}$ Therefore, for each neuron, orientation tuning profiles were obtained using LC and $\mathrm{HC}$ stimuli presented to the CRF alone and the CRF+ECS. Corresponding tuning profiles from each of the four conditions were then fit using a Gaussian model to determine the HWHH [Eq. (1)].

Examples for three domain neurons (top three rows) and three pinwheel neurons (bottom three rows) are given in Fig. 3. For both neuron types, when the CRF stimulus contrast was increased, tuning width broadened (Fig. 3, columns 1 and 2 ), demonstrating contrast variance. For example, the HWHH of the domain neuron in the first row increases from $25.1 \mathrm{deg}$ [Fig. 3(a)] to 30.3 deg [Fig. 3(b)], and tuning width of the pinwheel neuron in the fifth row increases from $23.2 \mathrm{deg}$ [Fig. 3(q)] to $35.7 \mathrm{deg}$ [Fig. 3(r)], representing a $21 \%$ increase for the domain cell and a $54 \%$ increase for the pinwheel neuron. Therefore, both cell types exhibit contrast variance. In these two examples, the pinwheel and domain neuron have similar

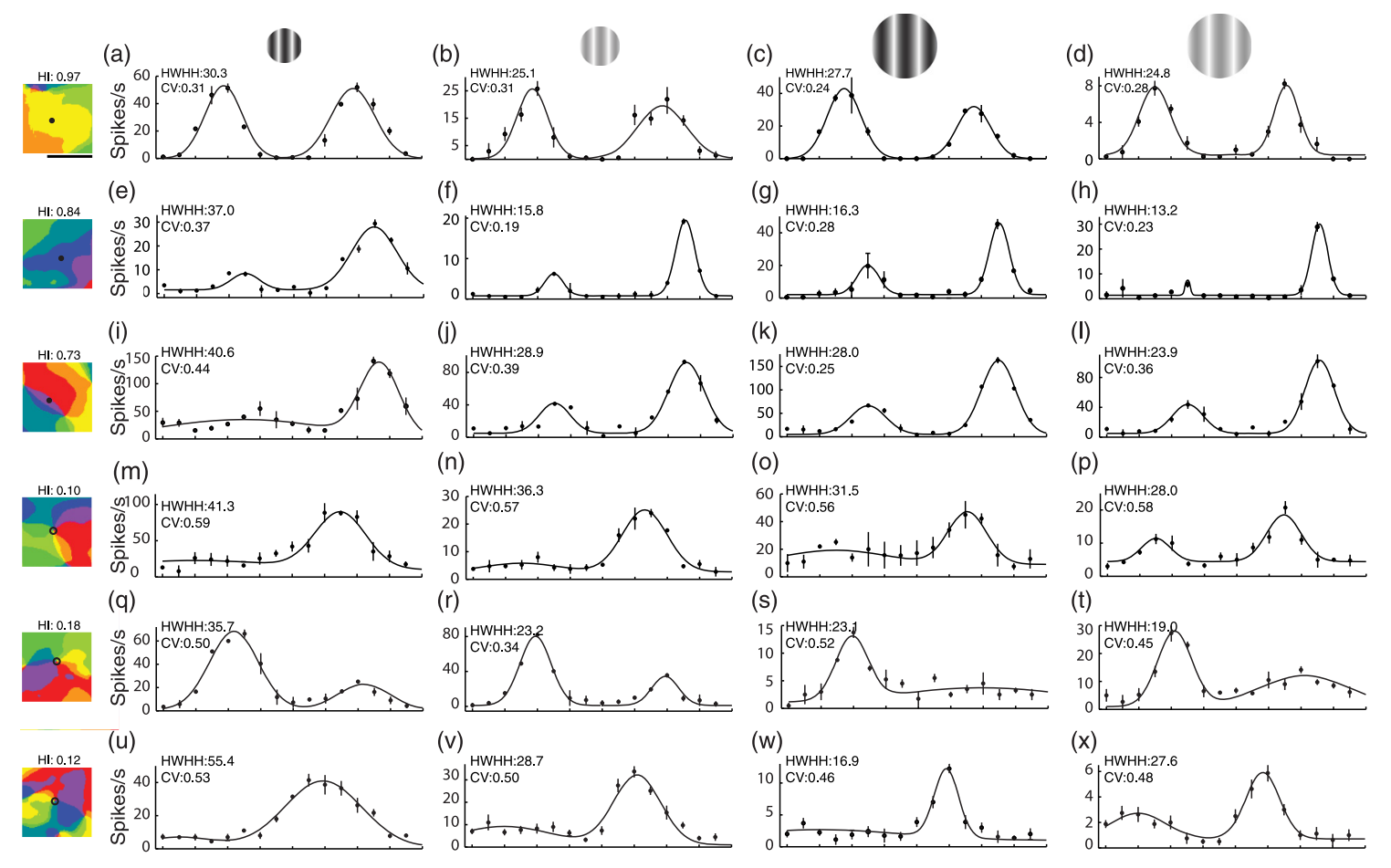

Fig. 3 Orientation tuning of representative $\mathrm{V} 1$ neurons relative to contrast, size, and location in the $\mathrm{V} 1$ orientation map. Orientation tuning curves are shown for six example neurons, three domain cells (a-d, e-h, and $\mathrm{i}-\mathrm{l})$, and three pinwheel cells $(\mathrm{m}-\mathrm{p}, \mathrm{q}-\mathrm{t}$, and $\mathrm{u}-\mathrm{x})$, in response to four stimulus conditions: HC CRF (a, e, i, m, q, and u; column 1), LC CRF (b, f, j, n, r, and v; column 2), HC CRF+ECS (c, g, k, o, s, and w; column 3), and LC CRF+ECS (d, h, I, p, t, and $x$; column 4). For these example cells, tuning width is broadest for the HC CRF condition (column 1). For the other three conditions (columns 2 to 4 ), tuning is sharper and relatively similar within cells (across each row). The largest differences in tuning width between domain and pinwheel cells are found for the HC CRF condition (column 1). Tuning width is measured as the $\mathrm{HWHH}$ as indicated in the left column, and corresponding values are given. CV values are also given for comparison. To the left of each tuning curve row, the recording locations are shown (black/white circle) in the center of $375-\mu \mathrm{m}^{2}$ regions of the $\mathrm{V} 1$ orientation map. The orientation selectivity of the local environment (180-deg radius) is quantified by a HI. Scale bar $=250 \mu \mathrm{m}$. 
orientation tuning curves at LC. At HC, tuning width broadens for both cells. However, the pinwheel neuron broadens substantially more, more than double the percent-increase of the domain neuron.

The population data closely reflect this pinwheel/domain difference in contrast variance seen in the example cells. The average percent-increase in tuning width for domain neurons was $30 \%$, from $23.3 \pm 1.5 \mathrm{deg}$ at $\mathrm{LC}$ to $30.3 \pm 1.8 \mathrm{deg}$ at $\mathrm{HC}$ [ $p<0.01$; Fig. 4(a), filled circles]. The average increase for pinwheel cells was larger, $41 \%$, from $24.9 \pm 1.2 \mathrm{deg}$ at $\mathrm{LC}$ and $35.1 \pm 1.5 \mathrm{deg}$ at $\mathrm{HC}[p<0.001$; Fig. 4(a), open circles $]$. As a result, while domain and pinwheel neurons both show significant contrast variance of orientation tuning, the broadening for

(a)
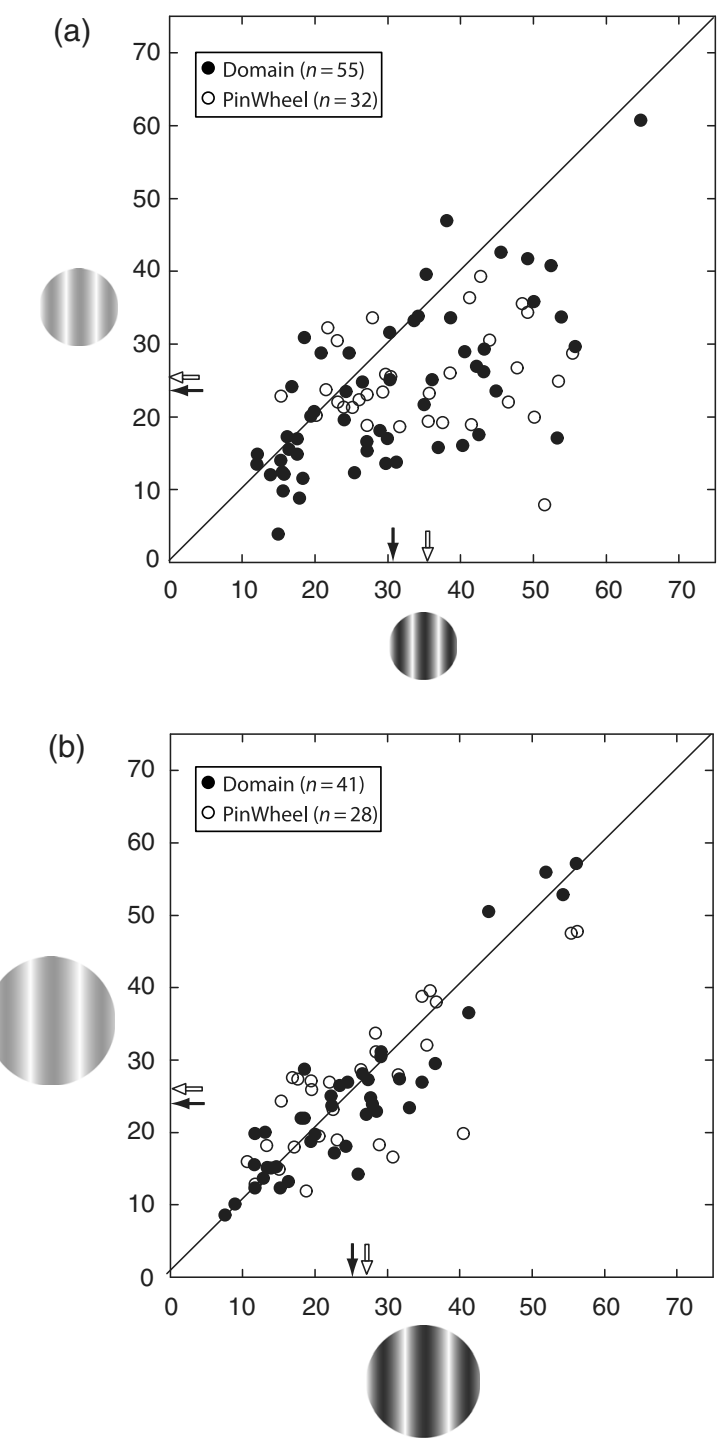

Fig. 4 Orientation tuning width varies with contrast for both pinwheel and domain neuron population when using optimally sized CRF stimuli but is contrast invariant when stimuli include the ECS. Scatter plots show the effects of contrast on HWHH. (a) For the CRF condition, tuning width is broader at $\mathrm{HC}(x$-axis) for both domain (filled circles) and pinwheel (open circles) neurons. (b) For the CRF + ECS condition, there is little difference in HWHH at low ( $y$-axis) and $\mathrm{HC}$, as both pinwheel and domain cells are found relatively close to the unity line. Note that the neurons plotted here are a subset of the neurons plotted in Fig. 5, because not all cells were measured at $\mathrm{HC}$ and LC for the same stimulus size. pinwheel neurons is greater. This difference is significant as seen through comparisons at $\mathrm{HC}$ where the tuning width is $35.2 \pm 1.5 \mathrm{deg}$ for pinwheel neurons and $29.9 \pm 1.5 \mathrm{deg}$ for domain neurons $[p<0.01$; Figs. 5(a) and 5(c)]. In Fig. 5, pinwheel and domain neurons are classified based on their HI, that is, the homogeneity of orientation preference in each neuron's local surroundings [ $\leq 180 \mu \mathrm{m}$; see Eq. (3)]. Again, for LC CRF, there is no significant difference in tuning [Figs. 5(b) and 5(d)]. We therefore conclude that CRF tuning width differences between domain and pinwheel cells depend on HC.

For larger visual stimuli, including the ECS, smaller changes in the orientation tuning width were generally observed when increasing from LC to HC for both domain and pinwheel neurons. This is shown by the examples in Fig. 3 (columns 3 and 4). The domain cell in row 1 shows an increase from $24.8 \mathrm{deg}$ [Fig. 3(c)] to $27.7 \mathrm{deg}$ [Fig. 3(d)] and the pinwheel neuron in row five shows an increase from $19.0 \mathrm{deg}$ [Fig. 3(s)] to $23.1 \mathrm{deg}$ [Fig. 3(t)]. Overall, no significant difference was found in the population data, where the average tuning widths at LC and HC were nearly identical, $24.5 \pm 1.8 \mathrm{deg}$ and $24.9 \pm$ $1.7 \mathrm{deg}$ for domain neurons and $26.1 \pm 1.9 \mathrm{deg}$ and $26.2 \pm 2.2 \mathrm{deg}$ for pinwheel neurons [Fig. 4(b)]. Therefore, orientation tuning is contrast invariant for large stimuli that include the ECS, as previously shown. ${ }^{36}$ Furthermore, as evidenced from the HI population distribution, there is also no significant difference in tuning width between domain and pinwheel cells for the CRF+ECS at either contrast [Figs. 5(e)-5(h)]; this is similar to the results for the LC CRF condition [Figs. 5(b) and 5(d)].

To summarize our results up to this point, we find that differences in HWHH between domain and pinwheel neurons are a result of contrast variance induced by $\mathrm{HC} \mathrm{CRF}$ stimuli, which broaden pinwheel neurons to a greater degree. Conversely, for all other conditions where orientation tuning is sharper for both cell types, either due to lower contrast or inclusion of the ECS, tuning widths are the same for domain and pinwheel neurons.

The HWHH is a better measure of local tuning features as orientations nearest to the preferred have the greatest impact on tuning width. Conversely, CV is a better measure of global tuning as responses at all orientations are factored equally [see Eq. (4)]. Factors such as a uniform increase in firing rate across all orientations can have a profound impact on $\mathrm{CV}$, but little impact on $\mathrm{HWHH}$, and resulting values from the two measures do not always correlate. ${ }^{54}$

We previously found that $\mathrm{CV}$ (or its inverse, orientation selectivity) was higher for pinwheel cells compared to domains at both HC and LC. ${ }^{28}$ These measurements were done only for the CRF. This pinwheel/domain difference for global tuning (CV) at LC [see Figs. 6(b) and 6(d)] was not seen here for local tuning [HWHH; Figs. 5(b) and 5(d)]. This suggests that LC stimuli affect orientation processing more globally than locally.

To compare with previous work, we also calculated CV here for CRF+ECS stimuli (see example CV values in Fig. 2). Consistent with our previous results, ${ }^{28}$ we find for the CRF that average $\mathrm{CV}$ is significantly higher for pinwheel compared to domain neurons at $\mathrm{HC}[0.40 \pm 0.02$ versus $0.53 \pm 0.02$; $p<0.001$; Figs. 6(a) and 6(c)] and for LC $[0.41 \pm 0.03$ versus $0.56 \pm 0.03 ; p<0.001$; Figs. $6(\mathrm{~b})$ and $6(\mathrm{~d})]$. We also find that for the CRF+ECS the average CV of pinwheel cells is higher than domain cells, but to a lesser degree and not statistically significant at either or high $[0.41 \pm 0.02$ versus $0.48 \pm 0.03$; 

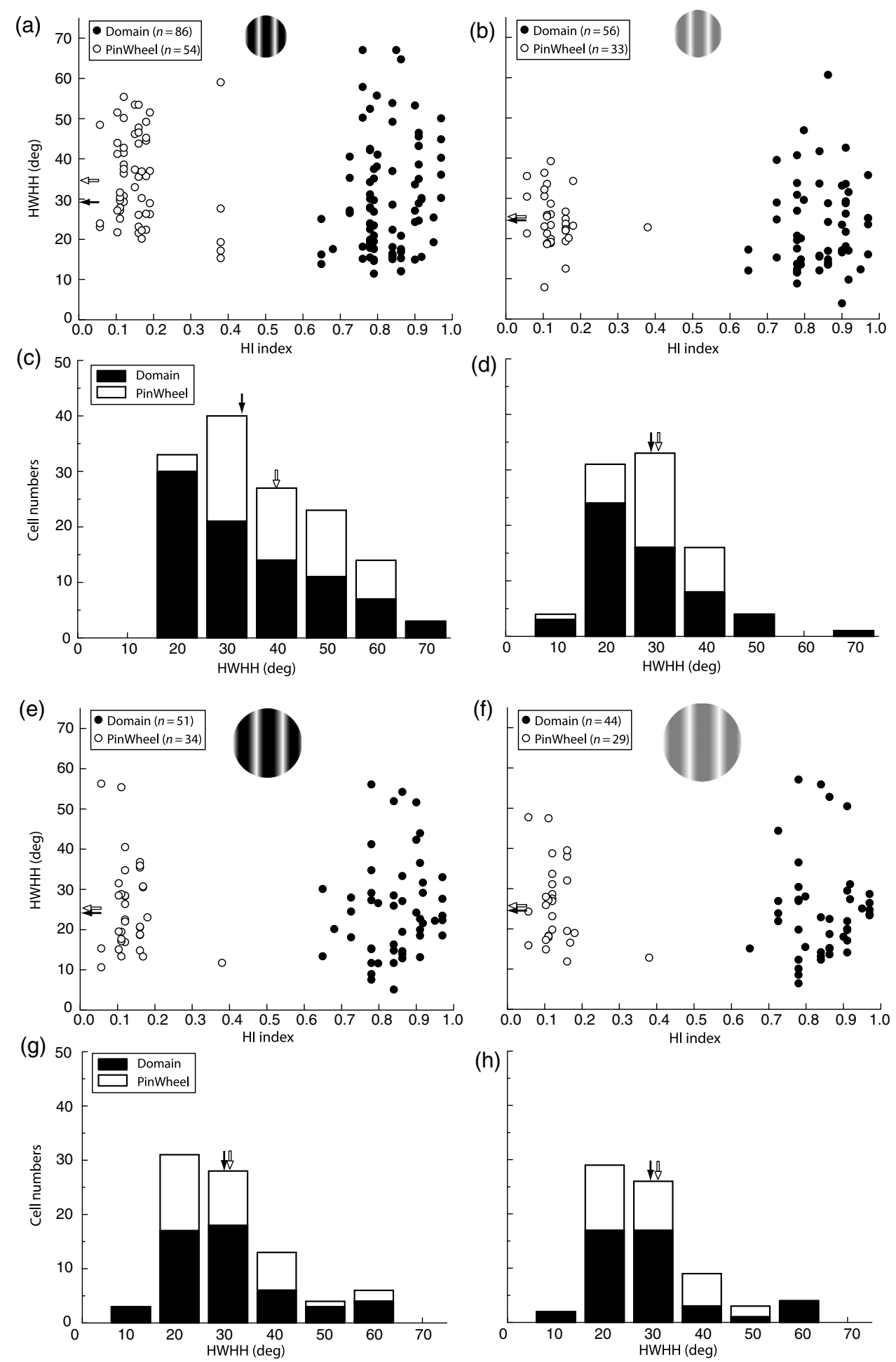

Fig. 5 Population orientation tuning width of the pinwheel neurons is broader than domain neurons only under the HC CRF condition. Scatter plots show the relationship between the HWHH ( $y$-axis) and $\mathrm{HI}$ ( $x$-axis) for the HC CRF (a) and CRF+ECS (e) and the LC CRF (b) and CRF+ECS (f). Low HI values correspond to neurons in pinwheels (open circles) and have an $\mathrm{HI}$ range of 0.05 to 0.38 ; Higher $\mathrm{HI}$ values correspond to domain neurons (filled circles) and range from 0.65 to 0.97 . Mean $\mathrm{HWHH}$ is indicated for domain and pinwheel neurons by black and white filled arrowheads, respectively. To facilitate comparisons, the number of cells per $\mathrm{HWHH}$ is presented in the bar graph in (c, $\mathrm{d}$ and $\mathrm{g}, \mathrm{h}$ ); black bars represent domain neurons, white bars represent pinwheel neurons. Arrows also indicate means for each cell type. The HC CRF $(a, c)$ is the only condition where the average HWHH differs significantly $(p<0.01)$ between domain (29.8 deg $\pm 1.5 \mathrm{deg})$ and pinwheel (35.2 deg $\pm 1.5 \mathrm{deg})$ neurons. 
(a)

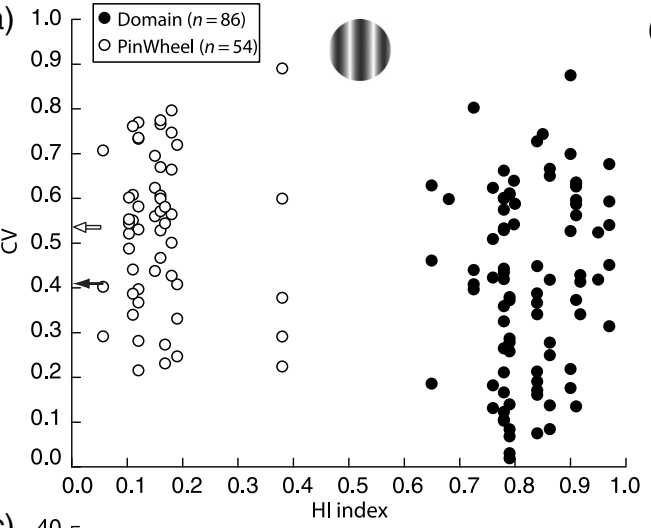

(c)

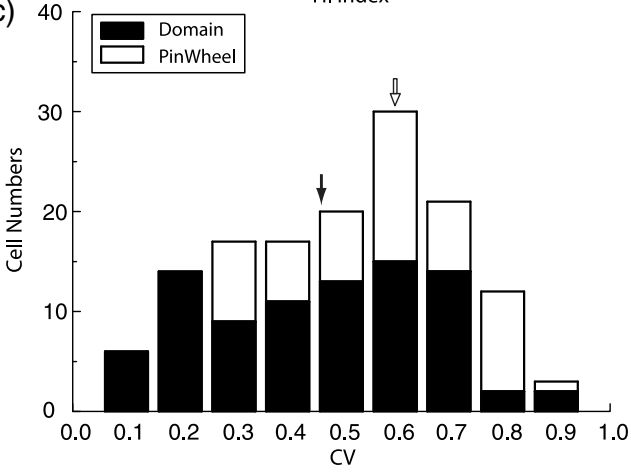

(b)

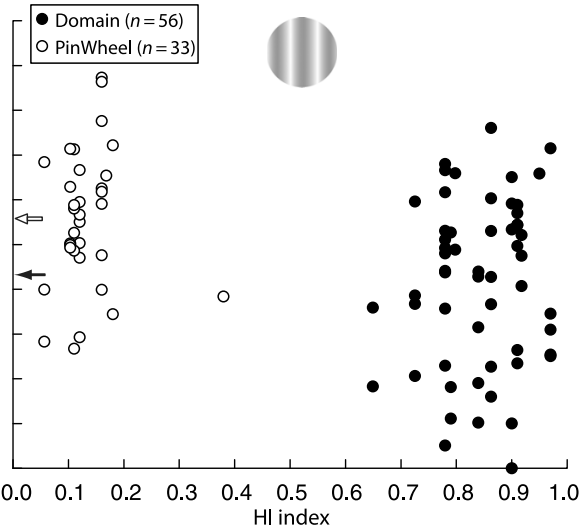

(d)

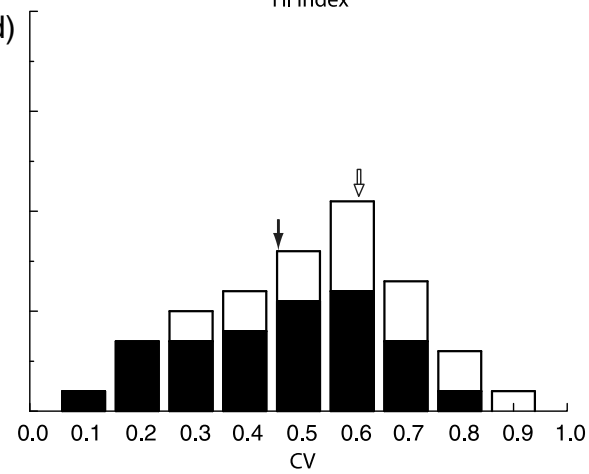

(e)

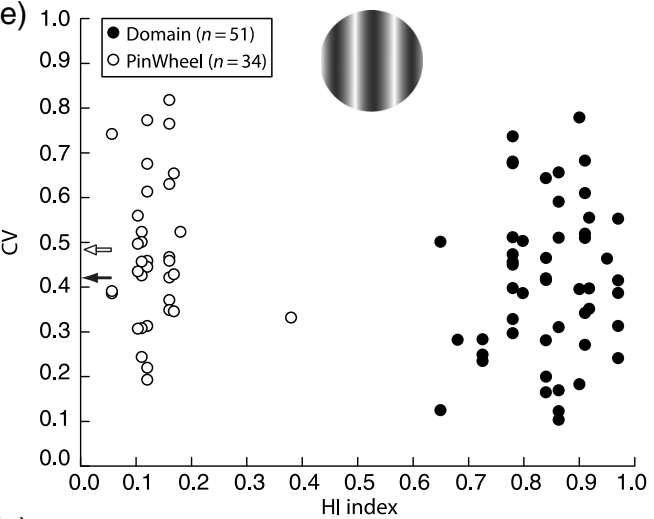

(g)

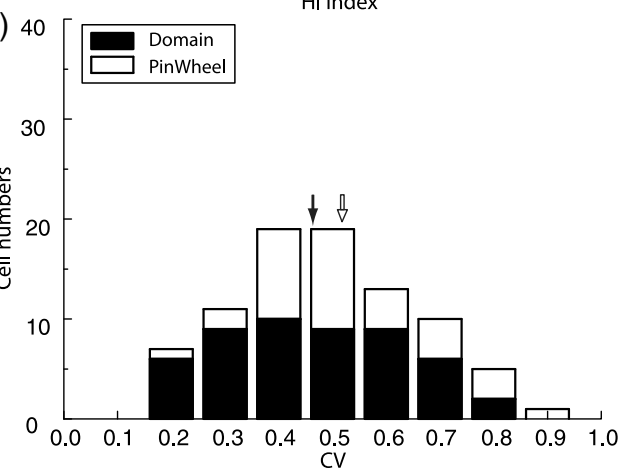

(f)

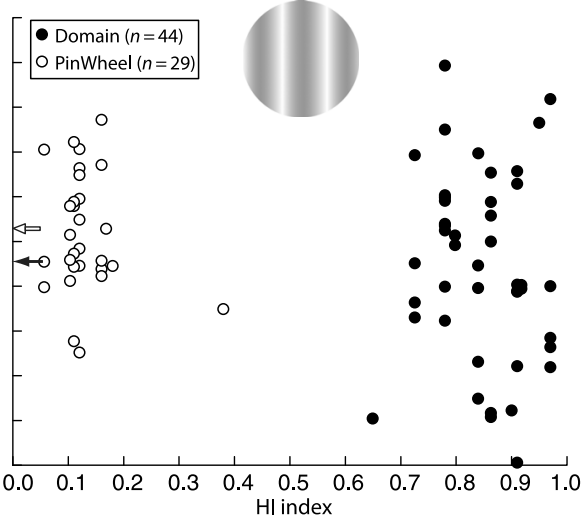

(h)

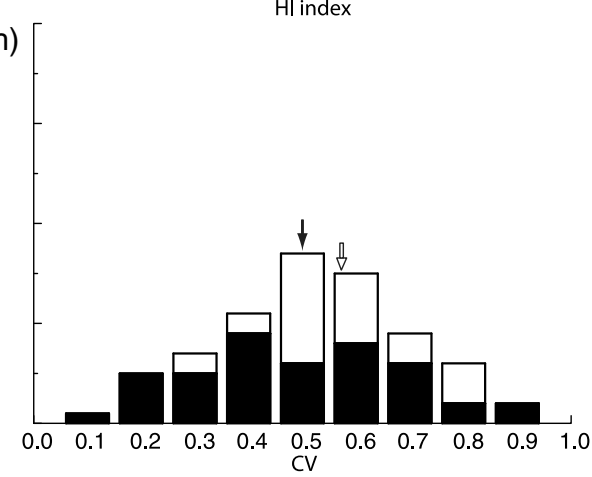

Fig. 6 Population CV of the pinwheel neurons is generally higher than domain neurons under multiple stimulus conditions. Scatter plots show the relationship between $\mathrm{CV}(y$-axis) and $\mathrm{HI}(x$-axis) for the $\mathrm{HC}$ CRF (a) and CRF+ECS (e) and the LC CRF (b) and CRF+ECS (f). Mean HWHH is indicated for domain and pinwheel neurons by black and white filled arrowheads, respectively. To facilitate comparisons, the number of cells at each $\mathrm{CV}$ is presented in the bar graph in (c, $d$ and $\mathrm{g}, \mathrm{h}$ ). The CRF condition results in significantly higher average $\mathrm{CV}$ for pinwheel compared to domain neurons at both high (a, $\mathrm{c} ; 0.40 \pm 0.02$ versus $0.53 \pm 0.02 ; p<0.001)$ and low $(b, d ; 0.41 \pm 0.03$ versus $0.56 \pm 0.03 ; p<0.001)$ contrast. Higher average $\mathrm{CV}$ for pinwheel neurons is also found for the CRF+ECS at high $(\mathrm{e}, \mathrm{g})$ and low $(\mathrm{f}, \mathrm{h})$ contrast, but the difference with domain neurons is not significant (see Sec. 3). 
$p=0.13$; Figs. 6(e) and 6(g) $]$ or low $[0.45 \pm 0.03$ versus $0.53 \pm 0.03 ; p=0.06$; Figs. $6(\mathrm{f})$ and $6(\mathrm{~h})]$ contrast. Therefore, as for the local measure of orientation tuning (HWHH), the more global measure (CV) may also depend on only the CRF being stimulated to reveal differences between domain and pinwheel neurons. But, in the case of $\mathrm{CV}$, domain and pinwheel differences are present at both $\mathrm{HC}$ and LC, suggesting that LC stimuli affect orientation processing more globally than locally, and reinforcing the idea that CV and HWHH do not always correlate.

\section{Discussion}

Our results provide new evidence that differences in orientation tuning for V1 neurons at different locations in the orientation preference map, domain versus pinwheel, depend on HC CRF conditions, which elicit the maximum response of a cell. This is the condition where long-range cortical circuits are the least engaged, ${ }^{32,33,36}$ suggesting that the differences may be mediated primarily through feedforward and/or local cortical processes where for pinwheel neurons the orientation preference of surrounding local inputs cover a much broader range compared to domain neurons. ${ }^{24,39}$ Conversely, all other conditions are thought to have reduced feedforward drive for LC and engage longer range cortical circuits, such as for LC summation that occurs from LC CRF stimuli or for surround suppression typically resulting from the ECS at either contrast. ${ }^{28,32-37,55,56}$ This suggests that tuning width differences between pinwheel and domain neurons are likely generated through more local cortical circuits before they can be equalized by longer range modulatory cortical inputs.

These results tie in with our recent report showing that larger stimuli extending beyond the CRF is key to contrast invariance, ${ }^{36}$ where orientation tuning widths at $\mathrm{HC}$ and $\mathrm{LC}$ do not change. ${ }^{53,57-59}$ Similarly, here we find that extending beyond the CRF is important for keeping the tuning widths comparable between neurons found at different orientation map locations. In both our previous study ${ }^{36}$ and this study, CRF+ECS results in similar orientation tuning widths, that is, no change at different contrasts and no difference between domain and pinwheel neurons. Inclusion of the ECS will typically reduce response amplitude ${ }^{35,43,60-63}$ and significantly sharpen tuning widths. ${ }^{36,38,40,41}$ This response reduction and sharpening of orientation tuning have been shown to result through a subtractive suppression. ${ }^{42}$ This suppression is likely mediated, at least in part, by longrange cortical circuits that synapse onto local inhibitory neurons. $22,64-67$

Previous studies comparing the tunings of pinwheel and domain neurons have been contradictory. Our results, in which tuning differences are only found using HC CRF, could at least partially explain the discrepancies. For example, Maldonado et al., ${ }^{23}$ and Mariño et al., ${ }^{24}$ both found pinwheel neurons to be as orientation tuned or selective as domain neurons in cats. However, both studies used HC full-field gratings, which include the ECS (see below for discussion of CV). On the other hand, Nauhaus et al. ${ }^{31}$ showed in cats and monkeys that the closer a neuron was to a pinwheel center, the broader it was tuned. In this study, it was not specified how large the HC visual stimuli were, but that the stimuli were large enough to cover the receptive fields of cells distributed over a 4- $\mathrm{mm}^{2}$ electrode array. Since receptive field center and size do not vary considerably over $4 \mathrm{~mm}$ in cats or monkeys, ${ }^{32,68-70}$ the stimulus size used may have corresponded well with the CRF of a number of cells. In this way, the results compare with what we report for HC CRF.

On the other hand, Ikezoe et al. ${ }^{29}$ used $\mathrm{HC}$ stimuli large enough to incorporate the full ECS and found higher CV for neurons located closer to pinwheel centers in monkey V1. However, as discussed in Sec. 3, CV is a more global measure of orientation selectivity and does not always correspond to local tuning widths. ${ }^{54}$ Furthermore, we also show here in cats that $\mathrm{CV}$, not tuning width, is higher for pinwheel cells using the HC and LC large stimuli (CRF+ECS), but our results comparing 85 neurons or less only approached significance [ $p=0.13$, Fig. 5(c); $p=0.06$, Fig. 5(d)]. Ikezoe et al. ${ }^{29}$ used two-photon calcium imaging that allowed for comparison of over 200 neurons in a single-image frame and over 1100 neurons for the study, which generated highly significant differences. Conversely, our lack of a significant difference in $\mathrm{CV}$ between domain and pinwheel neurons using CRF+ECS is consistent with the results of Mariño et al., ${ }^{24}$ who also compared CV for large full-field stimuli.

\section{Disclosures}

The authors declare that they do not have any conflicts of interest.

\section{Acknowledgments}

The experiments were performed in the laboratory of the corresponding author. All authors contributed to the (1) conception and design of the experiments, (2) collection, analysis, and interpretation of the data, and (3) writing and revising of the paper. This work was partially supported by funding from the Whitehall Foundation, Grant No. 2009-12-44 (D.C.L).

\section{References}

1. A. Grinvald et al., "High-resolution functional optical imaging: from the neocortex to the eye," Ophthalmol. Clin. North Am. 17, 53-67 (2004).

2. A. Grinvald et al., "Functional architecture of cortex revealed by optical imaging of intrinsic signals," Nature 324, 361-364 (1986).

3. W. H. Bosking et al., "Orientation selectivity and the arrangement of horizontal connections in tree shrew striate cortex," J. Neurosci. 17, 2112-2127 (1997).

4. R. D. Frostig et al., "Large-scale organization of rat sensorimotor cortex based on a motif of large activation spreads," J. Neurosci. 28, 13274 13284 (2008).

5. C. Gias et al., "Retinotopy within rat primary visual cortex using optical imaging," NeuroImage 24, 200-206 (2005).

6. V. A. Kalatsky and M. P. Stryker, "New paradigm for optical imaging: temporally encoded maps of intrinsic signal," Neuron 38, 529-545 (2003).

7. P. M. Knutsen, C. Mateo, and D. Kleinfeld, "Precision mapping of the vibrissa representation within murine primary somatosensory cortex," Philos. Trans. R. Soc. London Ser. B 371, 1705 (2016).

8. P. Li et al., "A motion direction preference map in monkey V4," Neuron 78, 376-388 (2013)

9. G. B. Liu and J. D. Pettigrew, "Orientation mosaic in barn owl's visual Wulst revealed by optical imaging: comparison with cat and monkey striate and extra-striate areas," Brain Res. 961, 153-158 (2003).

10. D. C. Lyon et al., "Optical imaging reveals retinotopic organization of dorsal V3 in New World owl monkeys," Proc. Natl. Acad. Sci. U. S. A. 99, 15735-15742 (2002).

11. R. Malach, R. B. Tootell, and D. Malonek, "Relationship between orientation domains, cytochrome oxidase stripes, and intrinsic horizontal connections in squirrel monkey area V2," Cereb. Cortex 4, 151-165 (1994).

12. J. H. Marshel et al., "Functional specialization of seven mouse visual cortical areas," Neuron 72, 1040-1054 (2011). 
13. A. W. Roe, "Long-term optical imaging of intrinsic signals in anesthetized and awake monkeys," Appl. Opt. 46, 1872-1880 (2007).

14. S. D. Van Hooser et al., "Orientation selectivity without orientation maps in visual cortex of a highly visual mammal," J. Neurosci. 25, 19-28 (2005).

15. M. Y. Villeneuve, M. P. Vanni, and C. Casanova, "Modular organization in area 21a of the cat revealed by optical imaging: comparison with the primary visual cortex," Neuroscience 164, 1320-1333 (2009).

16. H. Yu et al., "The coordinated mapping of visual space and response features in visual cortex," Neuron 47, 267-280 (2005).

17. T. Bonhoeffer and A. Grinvald, "Iso-orientation domains in cat visual cortex are arranged in pinwheel-like patterns," Nature 353, 429-431 (1991).

18. T. Bonhoeffer and A. Grinvald, "The layout of iso-orientation domains in area 18 of cat visual cortex: optical imaging reveals a pinwheel-like organization,” J. Neurosci. 13, 4157-4180 (1993).

19. A. Das and C. D. Gilbert, "Distortions of visuotopic map match orientation singularities in primary visual cortex," Nature 387, 594-598 (1997).

20. A. Das and C. D. Gilbert, "Topography of contextual modulations mediated by short-range interactions in primary visual cortex," Nature 399, 655-661 (1999).

21. M. Levy et al., "The shape of dendritic arbors in different functional domains of the cortical orientation map," J. Neurosci. 34, 32313236 (2014).

22. Y. J. Liu et al., "Tracing inputs to inhibitory or excitatory neurons of mouse and cat visual cortex with a targeted rabies virus," Curr. Biol. 23, 1746-1755 (2013).

23. P. E. Maldonado et al., "Orientation selectivity in pinwheel centers in cat striate cortex," Science 276, 1551-1555 (1997).

24. J. Mariño et al., "Invariant computations in local cortical networks with balanced excitation and inhibition," Nat. Neurosci. 8, 194-201 (2005).

25. J. Schummers et al., "Dynamics of orientation tuning in cat V1 neurons depend on location within layers and orientation maps," Front. Neurosci. 1, 145-159 (2007).

26. J. Schummers, J. Marino, and M. Sur, "Synaptic integration by V1 neurons depends on location within the orientation map," Neuron 36, 969-978 (2002).

27. F. Sengpiel and T. Bonhoeffer, "Orientation specificity of contrast adaptation in visual cortical pinwheel centres and iso-orientation domains," Eur. J. Neurosci. 15, 876-886 (2002).

28. M. Hashemi-Nezhad and D. C. Lyon, "Orientation tuning of the suppressive extraclassical surround depends on intrinsic organization of V1," Cereb. Cortex 22, 308-326 (2012).

29. K. Ikezoe et al., "Relationship between the local structure of orientation map and the strength of orientation tuning of neurons in monkey V1: a 2-photon calcium imaging study," J. Neurosci. 33, 16818-16827 (2013).

30. Y. J. Liu, M. Hashemi-Nezhad, and D. C. Lyon, "Sharper orientation tuning of the extraclassical suppressive-surround due to a neuron's location in the V1 orientation map emerges late in time," Neuroscience 229, 100-117 (2013).

31. I. Nauhaus et al., "Neuronal selectivity and local map structure in visual cortex," Neuron 57, 673-679 (2008).

32. A. Angelucci et al., "Circuits for local and global signal integration in primary visual cortex," J. Neurosci. 22, 8633-8646 (2002).

33. A. Angelucci and K. Sainsbury, "Contribution of feedforward thalamic afferents and corticogeniculate feedback to the spatial summation area of macaque V1 and LGN," J. Comput. Neurol. 498, 330-351 (2006).

34. J. R. Cavanaugh, W. Bair, and J. A. Movshon, "Selectivity and spatial distribution of signals from the receptive field surround in macaque V1 neurons," J. Neurophysiol. 88, 2547-2556 (2002).

35. J. R. Cavanaugh, W. Bair, and J. A. Movshon, "Nature and interaction of signals from the receptive field center and surround in macaque V1 neurons," J. Neurophysiol. 88, 2530-2546 (2002).

36. Y. J. Liu, M. Hashemi-Nezhad, and D. C. Lyon, "Contrast invariance of orientation tuning in cat primary visual cortex neurons depends on stimulus size," J. Physiol. 593, 4485-4498 (2015).

37. M. P. Sceniak et al., "Contrast's effect on spatial summation by macaque V1 neurons," Nat. Neurosci. 2, 733-739 (1999).

38. D. Xing et al., "Effect of stimulus size on the dynamics of orientation selectivity in Macaque V1," J. Neurophysiol. 94, 799-812 (2005).
39. M. Stimberg et al., "The operating regime of local computations in primary visual cortex," Cereb. Cortex 19, 2166-2180 (2009).

40. G. Chen, Y. Dan, and C. Y. Li, "Stimulation of non-classical receptive field enhances orientation selectivity in the cat," J. Physiol. 564, 233-243 (2005).

41. G. H. Henry, B. Dreher, and P. O. Bishop, "Orientation specificity of cells in cat striate cortex," J. Neurophysiol. 37, 1394-1409 (1974).

42. M. Okamoto et al., "Surround suppression sharpens orientation tuning in the cat primary visual cortex," Eur. J. Neurosci. 29, 1035-1046 (2009).

43. Y. J. Liu, M. Hashemi-Nezhad, and D. C. Lyon, "Dynamics of extraclassical surround modulation in three types of V1 neurons," J. Neurophysiol. 105, 1306-1317 (2011).

44. C. Bardy et al., "'Top-down' influences of ipsilateral or contralateral postero-temporal visual cortices on the extra-classical receptive fields of neurons in cat's striate cortex," Neuroscience 158, 951-968 (2009).

45. Y. L. Liu et al., "Very long-range disynaptic V1 connections by layer 6 pyramidal neurons revealed by transneuronal tracing with rabies virus," Eye Brain 6, 45-56 (2014).

46. J. J. Nassi, S. G. Lomber, and R. T. Born, "Corticocortical feedback contributes to surround suppression in V1 of the alert primate," J. Neurosci. 33, 8504-8517 (2013).

47. L. Schwabe et al., "Contrast-dependence of surround suppression in Macaque V1: experimental testing of a recurrent network model," NeuroImage 52, 777-792 (2010).

48. C. Wang et al., "Feedback signals from cat's area 21a enhance orientation selectivity of area 17 neurons," Exp. Brain Res. 182, 479-490 (2007).

49. J. D. Pettigrew, M. L. Cooper, and G. G. Blasdel, "Improved use of tapetal reflection for eye-position monitoring," Invest. Ophthalmol. Vis. Sci. 18, 490-495 (1979).

50. E. H. Ratzlaff and A. Grinvald, "A tandem-lens epifluorescence macroscope: hundred-fold brightness advantage for wide-field imaging," J. Neurosci. Methods 36, 127-137 (1991).

51. G. G. Blasdel and G. Salama, "Voltage-sensitive dyes reveal a modular organization in monkey striate cortex," Nature 321, 579-585 (1986).

52. M. Carandini and D. Ferster, "Membrane potential and firing rate in cat primary visual cortex," J. Neurosci. 20, 470-484 (2000).

53. H. J. Alitto and W. M. Usrey, "Influence of contrast on orientation and temporal frequency tuning in ferret primary visual cortex," J. Neurophysiol. 91, 2797-2808 (2004).

54. D. L. Ringach, R. M. Shapley, and M. J. Hawken, "Orientation selectivity in macaque V1: diversity and laminar dependence," J. Neurosci. 22, 5639-5651 (2002).

55. J. B. Levitt and J. S. Lund, "The spatial extent over which neurons in macaque striate cortex pool visual signals," Vis. Neurosci. 19, 439-452 (2002).

56. C. Tailby et al., "Two expressions of 'surround suppression' in V1 that arise independent of cortical mechanisms of suppression," Vis. Neurosci. 24, 99-109 (2007).

57. J. S. Anderson et al., "The contribution of noise to contrast invariance of orientation tuning in cat visual cortex," Science 290, 1968-1972 (2000).

58. G. Sclar and R. D. Freeman, "Orientation selectivity in the cat's striate cortex is invariant with stimulus contrast," Exp. Brain Res. 46, 457-461 (1982).

59. B. C. Skottun et al., "The effects of contrast on visual orientation and spatial frequency discrimination: a comparison of single cells and behavior," J. Neurophysiol. 57, 773-786 (1987).

60. T. Akasaki et al., "Suppressive effects of receptive field surround on neuronal activity in the cat primary visual cortex," Neurosci. Res. 43, 207-220 (2002).

61. G. C. DeAngelis, R. D. Freeman, and I. Ohzawa, "Length and width tuning of neurons in the cat's primary visual cortex," J. Neurophysiol. 71, 347-374 (1994).

62. F. Sengpiel, A. Sen, and C. Blakemore, "Characteristics of surround inhibition in cat area 17," Exp. Brain Res. 116, 216-228 (1997).

63. G. A. Walker, I. Ohzawa, and R. D. Freeman, "Suppression outside the classical cortical receptive field," Vis. Neurosci. 17, 369-379 (2000).

64. B. Ahmed et al., "Polyneuronal innervation of spiny stellate neurons in cat visual cortex," J. Comput. Neurol. 341, 39-49 (1994).

65. J. C. Anderson et al., "Synaptic output of physiologically identified spiny stellate neurons in cat visual cortex," J. Comput. Neurol. 341, 16-24 (1994). 
66. J. C. Anderson and K. A. Martin, "The synaptic connections between cortical areas V1 and V2 in macaque monkey," J. Neurosci. 29, 11283 11293 (2009).

67. J. A. Hirsch and C. D. Gilbert, "Synaptic physiology of horizontal connections in the cat's visual cortex," J. Neurosci. 11, 1800-1809 (1991)

68. D. Y. Ts'o, C. D. Gilbert, and T. N. Wiesel, "Relationships between horizontal interactions and functional architecture in cat striate cortex as revealed by cross-correlation analysis," J. Neurosci. 6, 1160-1170 (1986).
69. R. J. Tusa, L. A. Palmer, and A. C. Rosenquist, "The retinotopic organization of area 17 (striate cortex) in the cat," J. Comput. Neurol. 177, 213-235 (1978)

70. D. C. Van Essen, W. T. Newsome, and J. H. Maunsell, "The visual field representation in striate cortex of the macaque monkey: asymmetries, anisotropies, and individual variability," Vision Res. 24, 429-448 (1984).

Biographies for the authors are not available. 